\title{
A One-Dimensional Model with Phase Transition
}

\author{
Michel Mendès France ${ }^{1}$ and Gérald Tenenbaum ${ }^{2}$ \\ 1 Département de Mathématiques, Université Bordeaux I, 351, Cours de la Libération, \\ F-33405 Talence Cedex, France \\ 2 Département de Mathématiques, Université Nancy 1, BP 239, \\ F-54506 Vandœuvre Cedex, France
}

Received July 4, 1992; in revised form October 5, 1992

Abstract. Two repellent particles are bound to occupy two among the $k_{n}+1$ adjacent sites $0=x_{0}^{(n)}<x_{1}^{(n)}<\ldots<x_{k_{n}}^{(n)}=1$, say $x_{q}^{(n)}, x_{q+1}^{(n)}$. Define the Hamiltonian $\mathscr{H}_{q}^{(n)}=-\ln \left(x_{q+1}^{(n)}-x_{q}^{(n)}\right)$ and the partition function

$$
Z(\beta, n)=\sum_{0 \leq q<k_{n}} \exp \left\{-\beta \mathscr{H}_{q}^{(n)}\right\}
$$

We discuss the behaviour of the function

$$
F(\beta)=\limsup _{n \rightarrow+\infty} \frac{\ln Z(\beta, n)}{\ln k_{n}},
$$

closely related to the free energy. We prove that the smallest real zero of $F(\beta)$ is equal to the fractal dimension of the system and that this number, when less than one, is a critical value where $F$ is not analytic.

\section{Introduction and Description}

In a previous article [2] which was purely number theoretical, we defined and discussed a notion of dimension of a system of points in the unit interval and applied it to the study of divisors. Our purpose here is to give a physical interpretation of our results in the form of a simple one-dimensional model where a phenomenon of phase transition occurs.

Consider two repelling particles which are bound to occupy adjacent sites among $k+1$ sites $0=x_{0}<x_{1}<\ldots<x_{k-1}<x_{k}=1$ on the unit interval.

Fig. 1

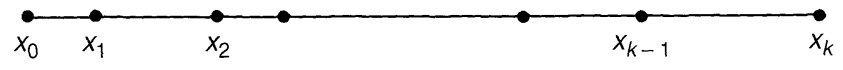


It is clear that the equilibrium state is attained when the two particles occupy the endpoints of one among the largest intervals $\left(x_{j}, x_{j+1}\right)$. Let us now describe the system in terms of statistical mechanics. A configuration $\sigma$ is one of the $k$ possible states $\left\{x_{\jmath}, x_{\jmath+1}\right\}$ and may well be represented by a finite sequence

$$
\sigma=(0,0, \ldots, 0,1,0, \ldots, 0)=0^{j} 10^{k-j+1},
$$

so that, in other words, $\sigma=\left\{\sigma_{q}\right\}_{q=0}^{q=k}$, where $\sigma_{q}=\delta_{q j}$. We define the potential energy (or Hamiltonian) of a configuration $\sigma$ by

$$
\mathscr{H}(\sigma):=-\sum_{q=0}^{k-1} \sigma_{q} \ln \left(x_{q+1}-x_{q}\right)=-\ln \left(x_{\jmath+1}-x_{j}\right) .
$$

According to the axioms of physics, the system reaches its equilibrium when the energy is minimal, i.e. when $\left(x_{j+1}-x_{j}\right)$ is maximal - a fact which was obvious from the beginning, as we already observed. This setting, however, enables us to develop further the statistical mechanics formalism with the introduction of an order-disorder parameter $\beta$ which plays the role of an inverse temperature, say $\beta=1 / T$, where $T$ may be thought of as the vibrational energy. If there is no vibration, the equilibrium is attained for minimal Hamiltonian; if however the vibrational energy tends to infinity, the various states $\left\{x_{j}, x_{\jmath+1}\right\}$ cannot be differentiated by the two particles and disorder prevails. Let us elaborate upon this statement.

One of the basic axioms in statistical mechanics is that the probability of a configuration $\sigma$ at temperature $T=1 / \beta$ is

$$
P_{\beta}(\sigma):=Z(\beta)^{-1} e^{-\beta . \mathscr{H}(\sigma)},
$$

where the normalizing factor, viz

$$
Z(\beta):=\sum_{\varrho} e^{-\beta \mathscr{H}(\varrho)},
$$

is the partition function. It is then obvious that, in the generic case when there is only one interval of maximal length, we have

$$
\lim _{\beta \rightarrow+\infty} P_{\beta}(\sigma)= \begin{cases}1 & \text { if } \mathscr{H}(\sigma) \text { is minimal } \\ 0 & \text { otherwise }\end{cases}
$$

Similarly, we have

$$
\lim _{\beta \rightarrow 0} P_{\beta}(\sigma)=1 / k
$$

Thus, we see that the system stays in its fundamental state at low temperature $(\beta \rightarrow+\infty)$ whereas at high temperature $(\beta \rightarrow 0)$ all possible states become equiprobable.

Following several authors (see e.g. [1 and 3], Chap. 6) we now allow negative temperatures. We then obtain

$$
\lim _{\beta \rightarrow-\infty} P_{\beta}(\sigma)= \begin{cases}1 & \text { if } \mathscr{H}(\sigma) \text { is minimal }, \\ 0 & \text { otherwise }\end{cases}
$$

if we assume that only one interval has minimal length. Hence, equilibrium occurs when the two particles are as close as possible. Thus, the system behaves as if the Hamiltonian had changed sign, that is to say that the particles now attract each other! 
The reader may be somewhat puzzled by the specific form for the Hamiltonian of our system. This choice originates in the fairly general observation that any evaluation of a Hausdorff dimension amounts to determining a change of behaviour in a sum of the type

$$
Z_{k}(\beta)=\sum_{0 \leq q<k} d_{q, k}^{\beta} \quad(k \rightarrow+\infty)
$$

and may therefore be also interpreted as a computation of a phase transition for a system with partition function $Z_{k}(\beta)$ - and hence with Hamiltonian $-\ln d_{q, k}$ for the $q^{\text {th }}$ configuration. The above system consisting of two repelling particles is certainly one of the simplest that yields a phase transition phenomenon. It is no doubt possible to extend some of the results presented here to systems of several repulsive particles, and we hope to develop this generalization in the future.

In our previous work [2], the study of sums of type $Z_{k}(\beta)$ was intended to exhibit the fractal nature of the set of divisors of a random integer and to determine its dimension. Thus negative $\beta$ had no significance, inasmuch as negative dimensions seem somewhat artificial. Here, on the contrary, $\beta$ appears as an inverse temperature and, since it is well known that negative temperatures do have a meaning in some systems $[1,3]$, the consideration of negative values is inevitable. Actually, the extension of our previous results to negative values of $\beta$ happens to be also interesting from the mathematical point of view.

\section{Thermodynamic Limit}

Here, we investigate the limiting behaviour of the system as the number of sites tends to infinity. Given an unbounded sequence of integers $\left\{k_{n}\right\}_{n=1}^{\infty}$, we let the system evolve as "time" $n$ increases. In the $n^{\text {th }}$ level of the system, the sites are

$$
0=x_{0}^{(n)}<x_{1}^{(n)}<\ldots<x_{k_{n}}^{(n)}=1,
$$

a configuration is a sequence $\sigma^{(n)}$ of $k_{n}-1$ zeros and ones, and the corresponding energy is

$$
\mathscr{H}\left(\sigma^{(n)}\right)=-\sum_{0 \leq q<k_{n}} \sigma_{q}^{(n)} \ln d_{q, n} \quad\left(d_{q, n}:=x_{q+1}^{(n)}-x_{q}^{(n)}\right) .
$$

The partition function then becomes

$$
Z(\beta, n):=\sum_{\sigma^{(n)}} \exp \left\{-\beta \mathscr{H}\left(\sigma^{(n)}\right)\right\}=\sum_{0 \leq q<k_{n}} d_{q, n}^{\beta} .
$$

In the thermodynamic limit, the free energy $\Psi(\beta)$ is given by the formula

$$
F(\beta):=-\beta \Psi(\beta)=\limsup _{n \rightarrow+\infty} \frac{\ln Z(\beta, n)}{\ln k_{n}} .
$$

Our main goal in this article is to describe the behaviour of $F(\beta)$ as $\beta$ varies from $-\infty$ to $+\infty$.

We summarize below some elementary facts concerning $F(\beta)$. This function is certainly bounded by 1 for $\beta \geq 0$ and we denote by $\beta^{*} \leq 0$ the infimum of the set of real numbers $\beta$ such that $F(\beta)$ is finite. 
Proposition 1. For $\beta>\beta^{*}$, the function $F(\beta)$ is finite, non-increasing and convex. It satisfies

$$
F(0)=1, F(1)=0, F(\beta) \geq-\beta\left(\beta>\beta^{*}\right), \quad F(\beta) \leq \max (0,1-\beta)(\beta \geq 0) .
$$

In particular, it is continuous, except perhaps at $\beta=\beta^{*}$.

Proof. Let $F(\beta, n):=(\ln Z(\beta, n)) / \ln k_{n}$. Then $F(\beta, n)$ is a decreasing function of $\beta$ which satisfies $F(0, n)=1, F(1, n)=0$. This plainly implies that $F(\beta)$ is nonincreasing as well as the first two relations in (1). To prove the third, let $d_{ \pm}(n)$ denote respectively the maximal and the minimal value of $d_{q, n}\left(0 \leq q<k_{n}\right)$, so that $d_{-}(n) \leq 1 / k_{n} \leq d_{+}(n)$. Then

$$
k_{n}^{-\beta} \leq \max \left(d_{-}(n)^{\beta}, d_{+}(n)^{\beta}\right) \leq Z(\beta, n) \quad(\beta \in \mathbb{R}),
$$

and, on letting $n \rightarrow+\infty$, we obtain the required inequality. For the last relation in (1), we may plainly restrict to the case $0 \leq \beta \leq 1$ because $F$ is non-increasing. The result is then implied by Hölder's inequality in the form

$$
Z(\beta, n)=\sum_{0 \leq q<k_{n}} d_{q, n}^{\beta} \leq\left(\sum_{0 \leq q<k_{n}} d_{q, n}\right)^{\beta}\left(\sum_{0 \leq q<k_{n}} 1\right)^{1-\beta}=k_{n}^{1-\beta}
$$

It remains to show that $F(\beta)$ is convex for $\beta>\beta^{*}$. This follows immediately from a new application of Hölder's inequality, namely

$$
\begin{aligned}
Z(r \beta+s \gamma, n) & =\sum_{0 \leq q<k_{n}} d_{q, n}^{r \beta+s \gamma} \\
& \leq\left(\sum_{0 \leq q<k_{n}} d_{q, n}^{\beta}\right)^{r}\left(\sum_{0 \leq q<k_{n}} d_{q, n}^{\gamma}\right)^{s}=Z(\beta, n)^{r} Z(\gamma, n)^{s}
\end{aligned}
$$

valid for all $\beta, \gamma$ in $\left(\beta^{*},+\infty\right)$ and all non-negative real numbers $r, s$ such that $r+s=1$. This evidently yields $F(r \beta+s \gamma) \leq r F(\beta)+s F(\gamma)$, which is all we need.

\section{Critical Temperatures}

It is clear from Proposition 1 that the set of real solutions $\beta$ to the equation $F(\beta)=0$ is an interval $\left[\beta_{-}, \beta_{+}\right]$, possibly reduced to a single point, and with the convention that $\beta_{+}$may be equal to $+\infty$. Since $F(0)=1, F(1)=0$, we certainly have

$$
0 \leq \beta_{-} \leq 1 \leq \beta_{+} \text {. }
$$

Moreover, as $F$ is non-increasing and convex, the condition $\beta_{-}<1$ implies $\beta_{+}=+\infty$. Actually, it is easy to note that $\beta_{+}$can only take the values 1 or $+\infty$. Indeed, if $\beta_{+}$is finite, then $F(\beta)=0$ for $\beta_{-} \leq \beta \leq \beta_{+}$, and this is compatible with the convexity of $F$ solely if $\beta_{-}=\beta_{+}=1$.

It is interesting to note that, if $\beta_{+}=+\infty$, then $F(\beta)$ cannot be analytic in the neighbourhood of $\beta_{-}$- and indeed, as we shall see in Sect. 5, $F(\beta)$ is not differentiable at $\beta_{-}$in most natural examples. Thus $\beta_{-}$appears in this case as a critical value, and we can say that our system has a phase transition at $\beta=\beta_{-}$. The following result gives a simple sufficient condition for the occurrence of a phase transition, in terms 
of the geometry of the system. We recall the definition of $d_{+}(n)$ from the proof of Proposition 1.

Proposition 2. Let $\delta:=\limsup _{n \rightarrow+\infty}\left(\ln d_{+}(n)\right) / \ln k_{n}$. Then, either

(i) $\delta=0$, and then $\beta_{+}=+\infty$,

or

(ii) $\delta<0$, and then $\beta_{-}=\beta_{+}=1$.

Proof. We plainly have, for all positive integers $n$ and all real numbers $\beta \geq 1$,

$$
d_{+}(n)^{\beta} \leq Z(\beta, n)=\sum_{0 \leq q<k_{n}} d_{q, n}^{\beta} \leq d_{+}(n)^{\beta-1} \sum_{0 \leq q<k_{n}} d_{q, n}=d_{+}(n)^{\beta-1} .
$$

Hence

$$
\beta \delta \leq F(\beta) \leq(\beta-1) \delta \quad(\beta \geq 1) .
$$

If $\delta=0$, it follows that $F(\beta)=0$ for all $\beta \geq 1$, whence $\beta_{+}=+\infty$. If $\delta<0$, then $F(\beta)<0$ for all $\beta>1$, so $\beta_{+} \leq 1$, and therefore $\beta_{+}=1$ by (2).

We note that in case (i) the quantity $\beta_{-}$need not be less than 1 . Examples with $\delta=0, \beta_{-}=1, \beta_{+}=+\infty$ are indeed easy to construct, e.g. $d_{q, n}=1 /(n \ln 2 n)$ $(0 \leq q<n), d_{n, n}=1-1 / \ln 2 n$.

\section{The Box Dimension Revisited}

In this section, we seek a characterization of the quantity $\beta_{-}$in terms of a natural notion of dimension that can be associated to systems of the type described above.

The dimension of a linear set $E$ is often defined in terms of coverings. If $N(\varepsilon)$ intervals are necessary to cover $E$, then

$$
\operatorname{dim} E:=\limsup _{\varepsilon \rightarrow 0} \frac{\ln N(\varepsilon)}{\ln (1 / \varepsilon)} .
$$

For our present purpose, we shall modify this definition. Given a system $X$ with levels $X_{n}:=\left\{x_{q}^{(n)}: 0 \leq q \leq k_{n}\right\}$, we cover each site $x_{q}^{(n)}$ by an interval of length $k_{n}^{-\alpha}$, centered at $x_{q}^{(n)}$ and put

$$
I_{q, n}(\alpha):=\left[x_{q}^{(n)}-\frac{1}{2} k_{n}^{-\alpha}, x_{q}^{(n)}+\frac{1}{2} k_{n}^{-\alpha}\right] \cap(0,1)
$$

Let

$$
\mu_{n}:=\operatorname{meas} \bigcup_{0 \leq q \leq k_{n}} I_{q, n}(\alpha)
$$

The number of intervals $I_{q, n}(\alpha)$ necessary to cover the set $X_{n}$ is approximately $\mu_{n} k_{n}^{\alpha}$. We then define

$$
d_{\alpha}(X):=\limsup _{n \rightarrow+\infty} \frac{\ln \left(\mu_{n} k_{n}^{\alpha}\right)}{\ln \left(k_{n}^{\alpha}\right)} .
$$

This plays the role of a dimension attached to the exponent $\alpha$. Since $\mu_{n} k_{n}^{\alpha}$ does not exceed the total number, $k_{n}$, of sites of $X_{n}$, we have

$$
0 \leq d_{\alpha}(X) \leq \min (1,1 / \alpha) \text {. }
$$


We define

$$
\operatorname{dim} X:=\sup _{\alpha>0} d_{\alpha}(X) .
$$

This reflects in a fairly sensitive way the geometry of the system, taking into account, in principle, all values of $\alpha$ - indeed, the function $\alpha \mapsto d_{\alpha}(X)$ is not necessarily nonincreasing, see Example 2, Sect. 5. In fact, we have the following characterization.

Theorem. For any system $X$, we have

$$
\beta_{-}=\operatorname{dim} X .
$$

In particular, we see that if $\beta_{+}$is infinite then the critical temperature is the inverse of the box dimension of the system.

The above theorem follows immediately from our previous work [2]. Indeed, we had defined there the dimension of $X$ by the formula

$$
\inf \left\{\beta: \limsup _{n \rightarrow+\infty} F(\beta, n)=0\right\},
$$

and formula (4) was our Théorème 1.

Of course, (5) gives a sufficient condition for the occurrence of a phase transition, namely that $\operatorname{dim} X<1$. But this is weaker than the condition $\delta=0$ of Proposition 2 . This is a consequence of implication (ii) of this statement. An alternative, direct proof rests on the following inequality, valid for all $\beta \leq 1$,

$$
Z(\beta, n)=\sum_{0 \leq q<k_{n}} d_{q, n}^{\beta} \geq d_{+}(n)^{\beta-1} \sum_{0 \leq q<k_{n}} d_{q, n}=d_{+}(n)^{\beta-1},
$$

whence

$$
F(\beta) \geq(\beta-1) \delta \quad(\beta \leq 1) .
$$

If $\operatorname{dim} X=\beta_{-}<1$, it follows on selecting $\beta=\beta_{-}$in (6) that $\delta \geq 0$, and hence $\delta=0$.

In this context, it is worthwhile to observe that the box dimension is invariant under a simple transformation such as replacing $X_{n}$ by $\frac{1}{2} X_{n} \cup\{1\}$, whereas the existence of a phase transition is certainly not, inasmuch as we must have $\delta=0$ for the transformed system.

\section{Examples}

We give here a number of representative examples of simple systems and discuss the occurrence of a phase transition.

Example 1. Smooth systems. Let $\gamma>0$. Define a system $X$ by $X_{n}=\left\{(q / n)^{\gamma}: 0 \leq\right.$ $q \leq n\}$ for all positive integers $n$. We call $X$ "smooth" because the sites are evenly distributed in $(0,1)$. As can be expected, a smooth system has dimension 1 . Nevertheless, there is generically a critical temperature. More precisely, we have

$$
Z(\beta, n)=\sum_{0 \leq q<n}\left(\frac{(q+1)^{\gamma}-q^{\gamma}}{n_{\gamma}}\right)^{\beta} \asymp \begin{cases}n^{1-\beta} & \text { if }(\gamma-1) \beta+1>0 \\ n^{-\gamma \beta} & \text { if }(\gamma-1) \beta+1 \leq 0\end{cases}
$$

where, here and in the sequel, $f \asymp g$ means that we have simultaneously $f=O(g)$ and $g=O(f)$. From the above estimate, we readily deduce that

$$
F(\beta)=\max (1-\beta,-\gamma \beta) .
$$



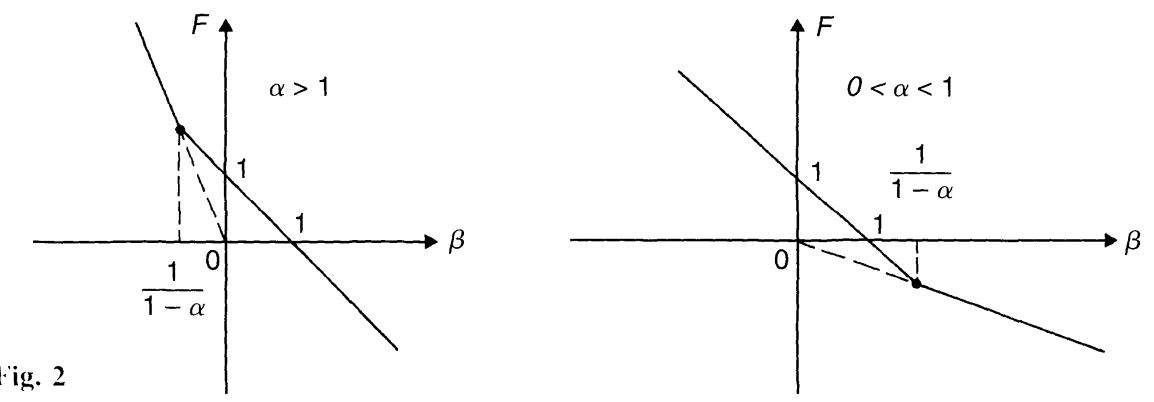

In particular, we see that $\operatorname{dim} X=1$ and that there is a critical point at $\beta=1 /(1-\gamma)$ whenever $\gamma \neq 1$. It is remarkable that this critical point is never at $\beta_{-}$. have

Finally, we note that a routine computation yields that for any smooth system we

$$
d_{\alpha}(X)=\min (1,1 / \alpha) \quad(\alpha>0)
$$

Thus, in view of (3), the function $\alpha \mapsto d_{\alpha}(X)$ is maximal for a smooth system.

Example 2. On the variations of $\alpha \mapsto d_{\alpha}(X)$. In most natural examples, $d_{\alpha}(X)$ is a non-increasing function of $\alpha$ and hence $\operatorname{dim} X=\lim _{\alpha \rightarrow 0+} d_{\alpha}(X)$. This is however by no means necessary, and we provide here an easy counter-example. Let $c>0$, and define $X$ by $X_{n}=\left\{q / n^{c+1}: 0 \leq q<n\right\} \cup\{1\}$. Then the number $T_{n}(\alpha)$ of intervals of length $n^{-\alpha}$ which are necessary to cover $X_{n}$ satisfies

$$
T_{n}(\alpha) \asymp \begin{cases}1 & \text { if } 0 \leq \alpha \leq c, \\ n^{\alpha-c} & \text { if } c<\alpha \leq c+1, \\ n & \text { if } \alpha>c+1\end{cases}
$$

Thus we obtain

$$
d_{\alpha}(X)= \begin{cases}0 & \text { if } 0 \leq \alpha \leq c \\ 1-c / \alpha & \text { if } c<\alpha \leq c+1 \\ 1 / \alpha & \text { if } \alpha>c+1\end{cases}
$$

In this case, $\operatorname{dim} X=1 /(c+1)$ corresponds to the unique maximum of $d_{\alpha}(X)$ at $\alpha=c+1$. Since $\operatorname{dim} X<1$, there is a critical point at $\beta_{-}=1 /(c+1)$; actually, it is easy to see that

$$
F(\beta)=\max (0,1-(c+1) \beta) .
$$

Example 3. The Cantor system. Let $\theta>2$. At level one, we consider the two intervals $[0,1 / \theta]$ and $[1-1 / \theta, 1]$ obtained by withdrawing from $[0,1]$ the middle interval of length $1-2 / \theta$. At level two, we form four intervals by withdrawing from each of our two previous intervals the middle open interval of length $\theta^{-1}(1-2 / \theta)$. We then carry on this procedure inductively. The Cantor system is defined as the system $X$ for which $X_{n}$ consists in the $2^{n+1}$ endpoints of the intervals constructed at level $n$. It can be shown (see [2]) that we then have

$$
\begin{aligned}
d_{\alpha}(X) & =\min \left\{\frac{\ln 2}{\ln \theta}, \frac{1}{\alpha}\right\} \quad(\alpha>0), \\
F(\beta) & =\max \left\{0,1-\beta \frac{\ln \theta}{\ln 2}\right\} \quad(\beta \in \mathbb{R}) .
\end{aligned}
$$


Fig. 3

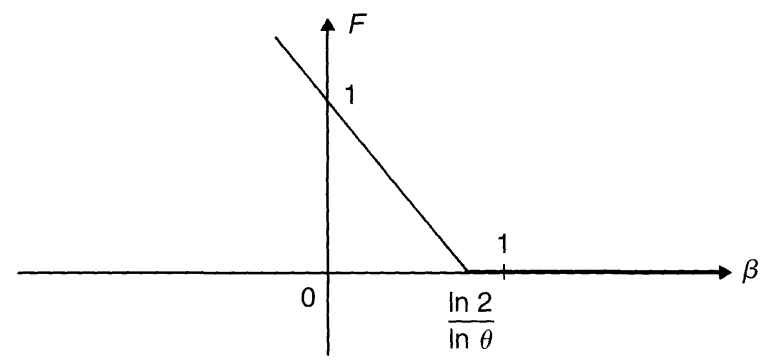

Hence, for the Cantor system, $\beta_{-}=\ln 2 / \ln \theta$ is a critical point.

Example 4. The divisor system. Let $n$ be a positive integer and $1=d_{1}<d_{2}<\ldots<$ $d_{\tau(n)}=n$ denote the increasing sequence of its divisors. Put

$$
X_{n}=\left\{\frac{\ln d_{q}}{\ln n}: 1 \leq q \leq \tau(n)\right\} .
$$

We have shown in [2] that there exists a subset $\mathbb{M} \subset \mathbb{N}$ of asymptotic density one such that the system $X=\left\{X_{m}\right\}_{m \in \mathbb{M}}$ satisfies

$$
\begin{aligned}
d_{\alpha}(X) & =\min \{\ln 2,1 / \alpha\} \quad(\alpha>0), \\
F(\beta) & =\max \{0,1-\beta / \ln 2\} \quad(\beta \in \mathbb{R}) .
\end{aligned}
$$

Thus $\beta_{-}=\ln 2$ is a critical point.

Example 5. A critical point where $F(\beta)$ is once but not twice differentiable. Define a system by selecting the differences $d_{q, n}\left(0 \leq q \leq k_{n}=2^{n}-1\right)$ in such a way that for every $j, 1 \leq j \leq n$ we have

$$
d_{q, n}=j^{n} / f(n)
$$

for exactly $2^{n-\jmath}$ values of $q$, where

$$
f(n)=\sum_{1 \leq j \leq n} j^{n} 2^{n-\jmath} .
$$

Put $\beta_{1}=\beta / \ln 2$. For given $n$, the function $t \mapsto t^{\beta n} 2^{n-t}(t \geq 1)$ is decreasing if $\beta<0$ and is otherwise unimodal, with a unique maximum at $t_{0}=\beta_{1} n$. Defining

$$
\beta_{2}:= \begin{cases}0 & \text { if } \beta<0 \\ \beta_{1} & \text { if } 0 \leq \beta \leq \ln 2 \\ 1 & \text { if } \beta>\ln 2\end{cases}
$$

we hence have

$$
\sum_{1 \leq j \leq n} j^{\beta n} 2^{n-j}=\left(1+\beta_{2} n\right)^{\beta n} 2^{n\left(1-\beta_{2}\right)} n^{O(1)} .
$$

This yields

$$
\begin{aligned}
Z(\beta, n) & =\sum_{0 \leq q<2^{n}} d_{q, n}^{\beta}=\frac{\sum_{1 \leq j \leq n} j^{\beta n} 2^{n-j}}{\left(\sum_{1 \leq j \leq n} j^{n} 2^{n-j}\right)^{\beta}} \\
& =\left(\frac{1}{n}+\beta_{2}\right)^{\beta n} 2^{n\left(1-\beta_{2}\right)} n^{O(1)}
\end{aligned}
$$


With the notation $Q(x):=x \ln x-x+1(x \geq 0)$, we obtain

$$
F(\beta)=\left\{\begin{array}{ll}
+\infty & \text { if } \beta<0, \\
Q\left(\beta_{2}\right) & \text { if } \beta \geq 0,
\end{array}= \begin{cases}+\infty & \text { if } \beta<0 \\
Q\left(\beta_{1}\right) & \text { if } 0 \leq \beta \leq \ln 2, \\
0 & \text { if } \beta>\ln 2\end{cases}\right.
$$

In particular, we see that $\beta_{-}=\ln 2$ is a critical point where $F$ is differentiable exactly once.

\section{References}

1. Landau, L., Lifchitz, E.: Physique mathématique, $2^{\text {nd }}$ ed. Moscow: Mir 1967

2. Mendès France, M., Tenenbaum, G.: Systèmes de points, diviseurs, et structure fractale. Bull. Soc. Math. de France 121 (1993)

3. Ramsey, N.F.: Thermodynamics and statistical mechanics at negative absolute temperatures. Phys. Rev. 103, 20 (1956)

Communicated by J. Fröhlich 
